\title{
Modelling Bacterial Metabolite Regulation of Vaginal Epithelial Cell Signaling in Bacterial Vaginosis
}

\author{
Alan Trinh ${ }^{1}$, Deva Chan², Doug Brubaker ${ }^{3}$ \\ ${ }^{1}$ Indiana University School of Medicine; ${ }^{2}$ Weldon School of Biomedical Engineering, Purdue \\ University; ${ }^{3}$ Regenstrief Center for Healthcare Engineering, Purdue University
}

\section{Background and Hypothesis}

Bacterial vaginosis, which is the imbalance of normal vaginal microbiota, contributes to preterm delivery, vaginitis, and decreased drug efficacy. Despite metronidazole efficacy in reducing BV contributing organisms, BV continues to recur in $50 \%$ of patients. Previous studies showing imidazole propionate's role in the pathogenesis of type II diabetes suggest that similar metaboliteregulated pathways in vaginal microbiomes may be the key in pathogenesis of uterine diseases such as BV. Thus, the purpose of this study was to observe the relationship between vaginal metabolites, host or microbiome-derived, and transcriptomic responses in vaginal epithelial tissues stratified by vaginal microbiome composition ("microbiome group"). The hypothesis was that differences in vaginal microbiome composition result in differential regulation of metabolitehost pathway functional relationships.

\section{Project Methods}

Transcript levels and metabolite concentrations precollected from 23 East African women were processed and analyzed via R. Transcriptomic data were converted into KEGG pathway enrichment scores via ssGSEA2.0, a package within R. Enrichment scores were correlated (Spearman) with metabolite levels by microbiome group and lactobacillus dominant phenotypes, and relationships were visualized via Heatmap3 and Cytoscape.

\section{Results:}

The results showed varying strengths in correlation among metabolites and KEGG pathway enrichment scores after filtering for strong correlations $(R>|0.5|)$ and significance $(p<0.05)$. Nonlactobacillus dominant microbiomes showed fewer strongly associated metabolite-KEGG pathway relationships compared to the lactobacillus dominant microbiome group, specifically the imidazole-related networks.

\section{Conclusion:}

In this study, variations in significant correlations among metabolites and KEGG pathways suggests that microbiome diversity may contribute to how metabolites regulate host pathways in vaginal epithelial cells. The reduced pathway interactions observed in imidazole compounds suggests that dysregulation may contribute to recurrence of bacterial vaginosis. This method of modelling could be used to characterize the regulation of critical pathways associated with the pathogenesis of bacterial vaginosis. 